\title{
Radial velocity variable, hot post-AGB stars from the MUCHFUSS project
}

\section{Classification, atmospheric parameters, formation scenarios}

\author{
N. Reindl ${ }^{1}$, S. Geier $2,3,4$, T. Kupfer ${ }^{5}$, S. Bloemen 6 , \\ V. Schaffenroth ${ }^{7,3}$, U. Heber ${ }^{3}$, B. N. Barlow ${ }^{8}$, and R. H. Østensen 9 \\ ${ }^{1}$ Institute for Astronomy and Astrophysics, Kepler Center for Astro and Particle Physics, Eberhard Karls University, Sand 1, \\ 72076 Tübingen, Germany \\ e-mail: reindl@astro.uni-tuebingen.de \\ 2 Department of Physics, University of Warwick, Coventry CV4 7AL, UK \\ ${ }^{3}$ Dr. Karl Remeis-Observatory \& ECAP, Astronomical Institute, Friedrich-Alexander University Erlangen-Nuremberg, \\ Sternwartstr. 7, 96049 Bamberg, Germany \\ 4 ESO, Karl-Schwarzschild-Str. 2, 85748 Garching bei München, Germany \\ 5 Division of Physics, Mathematics, and Astronomy, California Institute of Technology, Pasadena, CA 91125, USA \\ ${ }^{6}$ Department of Astrophysics/IMAPP, Radboud University Nijmegen, PO Box 9010, 6500 GL Nijmegen, The Netherlands \\ 7 Institute for Astro- and Particle Physics, University of Innsbruck, Technikerstr. 25/8, 6020 Innsbruck, Austria \\ 8 Department of Physics, High Point University, 833 Montlieu Avenue, High Point, NC 27262, USA \\ 9 Institute of Astronomy, KU Leuven, Celestijnenlaan 200D, 3001 Heverlee, Belgium
}

Received 24 October 2015 / Accepted 5 January 2016

\begin{abstract}
In the course of the MUCHFUSS project we recently discovered four radial velocity (RV) variable, hot $\left(T_{\text {eff }} \approx 80000-110000 \mathrm{~K}\right)$ post-asymptotic giant branch (AGB) stars. Among them, we found the first known RV variable $\mathrm{O}(\mathrm{He})$ star, the only second known RV variable PG 1159 close binary candidate, as well as the first two naked (i.e., without planetary nebula (PN)) H-rich post-AGB stars of spectral type $\mathrm{O}(\mathrm{H})$ that show significant RV variations. We present a non-LTE spectral analysis of these stars along with one further $\mathrm{O}(\mathrm{H})$-type star whose RV variations were found to be not significant. We also report the discovery of a far-infrared excess in the case of the PG 1159 star. None of the stars in our sample displays nebular emission lines, which can be explained well in terms of a very late thermal pulse evolution in the case of the PG 1159 star. The "missing" PNe around the $\mathrm{O}(\mathrm{H})$-type stars seems strange, since we find that several central stars of PNe have much longer post-AGB times. Besides the non-ejection of a PN, the occurrence of a late thermal pulse, or the re-accretion of the PN in the previous post-AGB evolution offer possible explanations for those stars not harbouring a PN (anymore). In the case of the $\mathrm{O}(\mathrm{He})$ star $\mathrm{J} 0757$, we speculate that it might have been previously part of a compact He transferring binary system. In this scenario, the mass transfer must have stopped after a certain time, leaving behind a low-mass close companion that may be responsible for the extreme RV shift of $107.0 \pm 22.0 \mathrm{~km} \mathrm{~s}^{-1}$ that was measured within only 31 min.
\end{abstract}

Key words. binaries: spectroscopic - stars: AGB and post-AGB - stars: evolution - stars: atmospheres

\section{Introduction}

The discovery and analysis of close binaries throughout all evolutionary stages plays an important role in various astronomical fields. Due to the radiation of gravitational waves, very close (periods of less than a few hours) binary systems may merge within a Hubble time. If the system contains two sufficiently massive white dwarfs or a subdwarf star and a sufficiently massive white dwarf, this evolutionary path can lead to a type Ia supernova (Webbink 1984), the so-called double degenerate scenario. If the total mass of the two merging stars does not exceed the Chandrasekhar limit, He-dominated stars, i.e., R Coronae Borealis stars (RCB), extreme helium (EHe) stars, He-rich subdwarf $\mathrm{O}(\mathrm{He}-\mathrm{sdO})$ stars or $\mathrm{O}(\mathrm{He})$ stars may be produced (Webbink 1984; Iben \& Tutukov 1984; Justham et al. 2011; Zhang \& Jeffery 2012b,a; Zhang et al. 2014; Reindl et al. 2014b). The existence of planetary nebulae (PNe) around every other $\mathrm{O}(\mathrm{He})$ star and the [WN] type central stars, however, put in question the possibility that all He-dominated stars have a merger origin. Those stars might have lost their H-rich envelope via enhanced mass loss during their post-asymptotic giant branch (AGB) evolution, which was possibly triggered by a close companion (Reindl et al. 2014b).

Close binary evolution is also needed to explain the formation of low-mass He-core white dwarfs $\left(M<0.5 M_{\odot}\right)$, because the evolutionary timescale of the supposed low-mass, single main-sequence progenitor stars significantly exceeds the Hubble time. The orbital energy that is released during a common envelope phase is supposed to rapidly expel the envelope of the pre-low-mass white dwarf and may terminate the growth of the He-core before it reaches a sufficient mass for He ignition (Paczynski 1976; Webbink 1984; Iben \& Tutukov 1986).

Also the formation of hot subdwarfs (sdO/B), located at the (post-) extreme horizontal branch, may best be understood in 
terms of close binary evolution. The progenitors of these stars are expected to have already undergone a strong mass-loss on the red giant branch (RGB), which was likely triggered by the close companion. If the mass-loss was sufficiently high, these stars leave the RGB and ignite He only while evolving to or descending the white dwarf cooling curve, and will consequently, reach a hotter zero-age horizontal position (Brown et al. 2001; Lanz et al. 2004).

Finally, close binaries are proposed to play a crucial role in the formation of asymmetrical $\mathrm{PNe}$, which make up the great majority (80-85\%, Parker et al. 2006) of all PNe. The so-called binary hypothesis (De Marco et al. 2009) states that a companion is needed to account for the non-spherical shapes of these PNe. Several research groups are trying to determine the frequency of binary central stars of PNe (CSPNe) and the properties of these systems. Most of the known close binary CSPNe were detected via periodic flux variability due to eclipses, irradiation, and/or ellipsoidal deformation (Bond 2000; Miszalski et al. 2009). This technique is, however, biased against binaries with periods longer than about two weeks, against binaries with low inclination angles, and against companions with small radii (De Marco et al. 2008). Large radial velocity (RV) surveys of CSPNe are telescope-time expensive and not always straightforward, because most of the strongest photospheric lines are blended with nebular emission lines, and the extraction of the pure stellar spectrum may often not be successful in the case of very compact nebulae.

Fortunately, several hundreds of hot subluminous stars, which generally do not display nebular lines, were observed in the course of the Sloan Digital Sky Survey (SDSS). Because SDSS spectra are co-added from at least three individual integrations (with a typical exposure time of $15 \mathrm{~min}$ ), they offer the ideal basis for the search for RV variations. The MUCHFUSS (Massive Unseen Companions to Hot Faint Underluminous Stars from SDSS) survey made use of that to search for hot subdwarf stars with massive compact companions (Geier et al. 2011). For that purpose, hot subdwarfs were selected from the SDSS DR7 by color (i.e., they were required to be point sources with $u-g<$ 0.4 and $g-r<0.1)$ and visual inspection of their spectra in order to distinguish them from hot white dwarfs or extragalactic sources. Those stars, whose SDSS sub-spectra revealed high $\mathrm{RV}$ variations, were selected as candidates for follow-up spectroscopy to derive the RV curves and the binary mass functions of the systems. In this way, more than $100 \mathrm{RV}$-variable subdwarfs have been found (Geier et al. 2015).

Hot post-AGB stars directly overlap with hot subdwarfs of O-type (sdO) in the color-color plane. If hot post-AGB stars are not surrounded by a compact nebula and/or do not suffer from still ongoing strong mass loss (i.e., weak emission line stars, Wolf Rayet type central stars, whose spectra show or are even dominated by emission lines), their spectra may look indistinguishable from the less luminous sdO stars. About three-quarters of all hot post-AGB stars display a normal H-rich composition and, in case their spectra are dominated by absorption lines, they can be classifed as $\mathrm{O}(\mathrm{H})$ or $\mathrm{hgO}(\mathrm{H})$ stars (the latter case applies to high gravity stars which are positioned on the white dwarf cooling track, Mendez 1991). Hot, H-deficient stars, which show predominatly absorption lines and lie in the post-AGB region, are either classified as $\mathrm{O}(\mathrm{He})$ stars (typically showing more than $90 \% \mathrm{He}$, by mass), or PG 1159 stars, whose spectra show besides $\mathrm{He}$ also strong lines of carbon. By eye, the spectra of $\mathrm{O}(\mathrm{H}), \mathrm{O}(\mathrm{He})$, and $\mathrm{PG} 1159$ stars may look very similar to those of the less luminous $\mathrm{sdO}, \mathrm{He}-\mathrm{sdO}$, and C-strong lined $\mathrm{He}$-sdO stars, respectively. Thus, the sample of Geier et al. (2015)

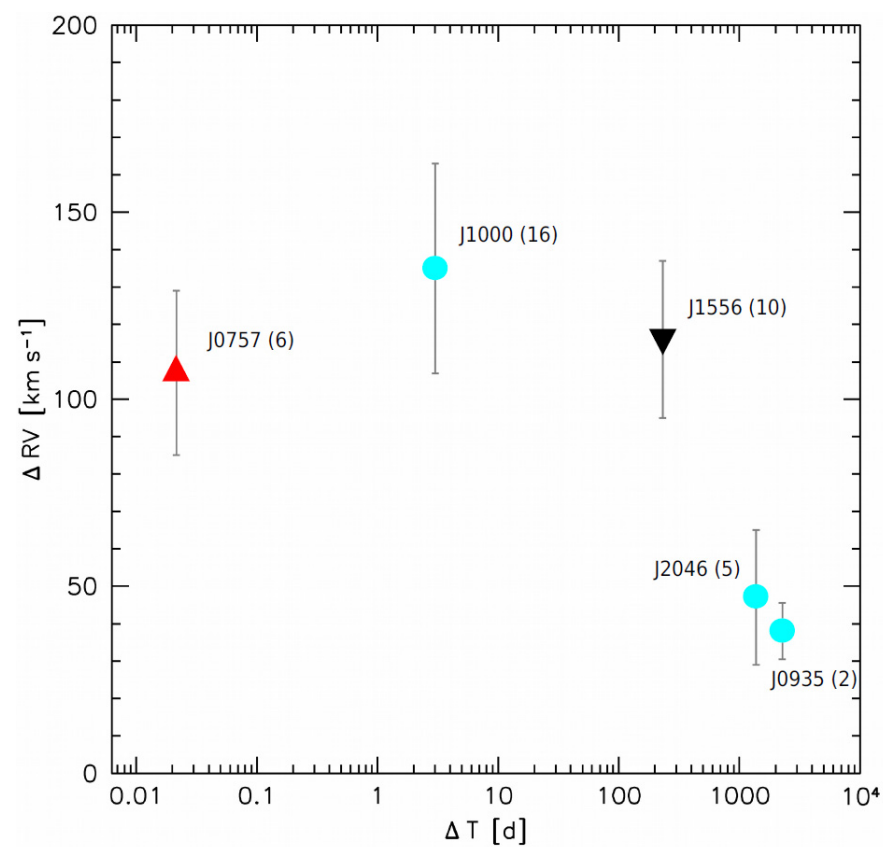

Fig. 1. Highest RV shift between individual spectra plotted against time difference between the corresponding observing epochs for the $\mathrm{O}(\mathrm{He})$ star J0757 (red triangle), the PG1159 star J1556 (black inverted triangle), and the three $\mathrm{O}(\mathrm{H})$ stars J1000, J2046, and J0935 (blue filled circles). The numbers in the brackets correspond to the number of epochs each star was observed. Note that the RV shifts of J2046 were found to be not significant.

also contains a small fraction of hot post-AGB candidates, namely SDSS J075732.18+184329.3 (hereafter J0757), SDSS J093521.39+482432.4 (J0935), SDSS J100019.98003413.3 (J1000), SDSS J155610.40+254640.3 (J1556), and SDSS J204623.12-065926.8 (J2046). Previous analysis of these stars indicates very high effective temperatures (Werner et al. 2014; Geier et al. 2015). Because of the rareness of such objects as well as their importance for our understanding of the late, hot stages of stellar evolution we decided to subject these five stars to a more comprehensive analysis and discussion of their evolutionary status.

The paper is organized as follows. First, we describe the observations and the discovery of RV variations (Sect. 2). Then, we determine the parameters of the stars in Sect. 3. In Sect. 4, there follows a search for a PN and an infrared excess. The evolutionary status of the stars is discussed in Sect.5, and finally we conclude in Sect. 6.

\section{Observations and detection of radial velocity variations}

Individual observations and RV measurements of the five stars are listed in Geier et al. (2015). The RVs were measured by fitting a set of mathematical functions (Gaussians, Lorentzians, and polynomials) to the spectral lines using the FITSB2 routine (Napiwotzki et al. 2004). The fraction of false detections produced by random fluctuations and the significance of the measured RV variations were calculated as described in Maxted et al. (2001).

In the spectra of J0757, J0935, J1000, and J1556 we discovered significant RV shifts, while the RV variations in the five SDSS sub-spectra of J2046 were found to be most likely caused by random fluctuations (false-detection probability $\mathrm{p}$ is larger than $0.01 \%$ ). In Fig. 1 we show their highest RV shift 
between individual spectra plotted against time difference between the corresponding observing epochs. Most notably, we discovered a maximum RV shift of $107.0 \pm 22.0 \mathrm{~km} \mathrm{~s}^{-1}$ within only $31 \mathrm{~min}$ in the six SDSS sub-spectra (taken on two consecutive nights) of J0757. The object J1000 was observed 16 times in the course of the SDSS and reveals a maximum RV shift of $135.0 \pm 28.0 \mathrm{~km} \mathrm{~s}^{-1}$. In addition to the nine SDSS observations of J1556, we obtained one medium-resolution spectrum with TWIN at the Calar Alto $3.5 \mathrm{~m}$ telescope and found a maximum RV shift of $116.0 \pm 21.0 \mathrm{~km} \mathrm{~s}^{-1}$. For J0935 second epoch spectroscopy was obtained with WHT/ISIS. The maximum RV shift is $38.0 \pm 7.5 \mathrm{~km} \mathrm{~s}^{-1}$.

To verify the close binary nature of the stars, we tried to derive orbital periods and RV semiamplitudes of the objects with more than ten epochs as described in Geier et al. (2015). Unfortunately, in both cases the RV datasets were insufficient to put any meaningful constraints on the orbital parameters.

\section{Stellar parameters and distances}

In this section we first perform a non-local thermodynamic equilibrium (non-LTE) spectral analysis to derive the effective temperatures, surface gravities, and the chemical composition of the five stars (Sect. 3.1). These parameters are then used in Sect. 3.2 to derive their masses, luminosities, and distances.

\subsection{Spectral analysis}

Quantitative spectral analysis is the key to derive the surface parameters of the stars and to understand their evolutionary status. Our spectral analysis was based on the SDSS observations of the stars. The single spectra have been corrected for their orbital motion and co-added. For the model calculations we employed the Tübingen non-LTE model-atmosphere package (TMAP ${ }^{1}$, Werner et al. 2003; Rauch \& Deetjen 2003) which allows plane-parallel, non-LTE, fully metal-line blanketed model atmospheres in radiative and hydrostatic equilibrium to be computed. Model atoms were taken from the Tübingen model atom database $\mathrm{TMAD}^{2}$. To calculate synthetic line profiles, we used Stark line-broadening tables provided by Barnard et al. (1969) for He I $\lambda \lambda 4026,4388,4471,4921 \AA$, Barnard et al. (1974) for He I $\lambda 4471 \AA$, and Griem (1974) for all other He I lines. For He II, we used the tables provided by Schöning \& Butler (1989), for H I tables provided by Tremblay \& Bergeron (2009), and for C IV tables provided by Dimitrijević et al. (1991) and Dimitrijević \& Sahal-Brechot (1992).

\subsubsection{H-rich stars}

The spectra of J0935, J1000, and J2046 are dominated by hydrogen Balmer lines. Spectral lines of He II are of moderate strength, but no He I lines can be detected. However, the signal to noise $(\mathrm{S} / \mathrm{N})$ of the observations is too poor $(S / N \approx 20-30)$ to exclude their presence from the outset. To derive effective temperatures, surface gravities, and He abundances of these stars, we calculated a model grid spanning from $T_{\text {eff }}=40000-140000 \mathrm{~K}$ (step size $1250 \mathrm{~K}$ ) and $\log g=4.75-6.5$ (step size $0.25 \mathrm{dex}$ ) for six different $\mathrm{He}$ abundances $(\mathrm{He}=0.1,0.2,0.3,0.4,0.5$,

\footnotetext{
1 http://astro.uni-tuebingen.de/ TMAP

2 http://astro.uni-tuebingen.de/ TMAD
}

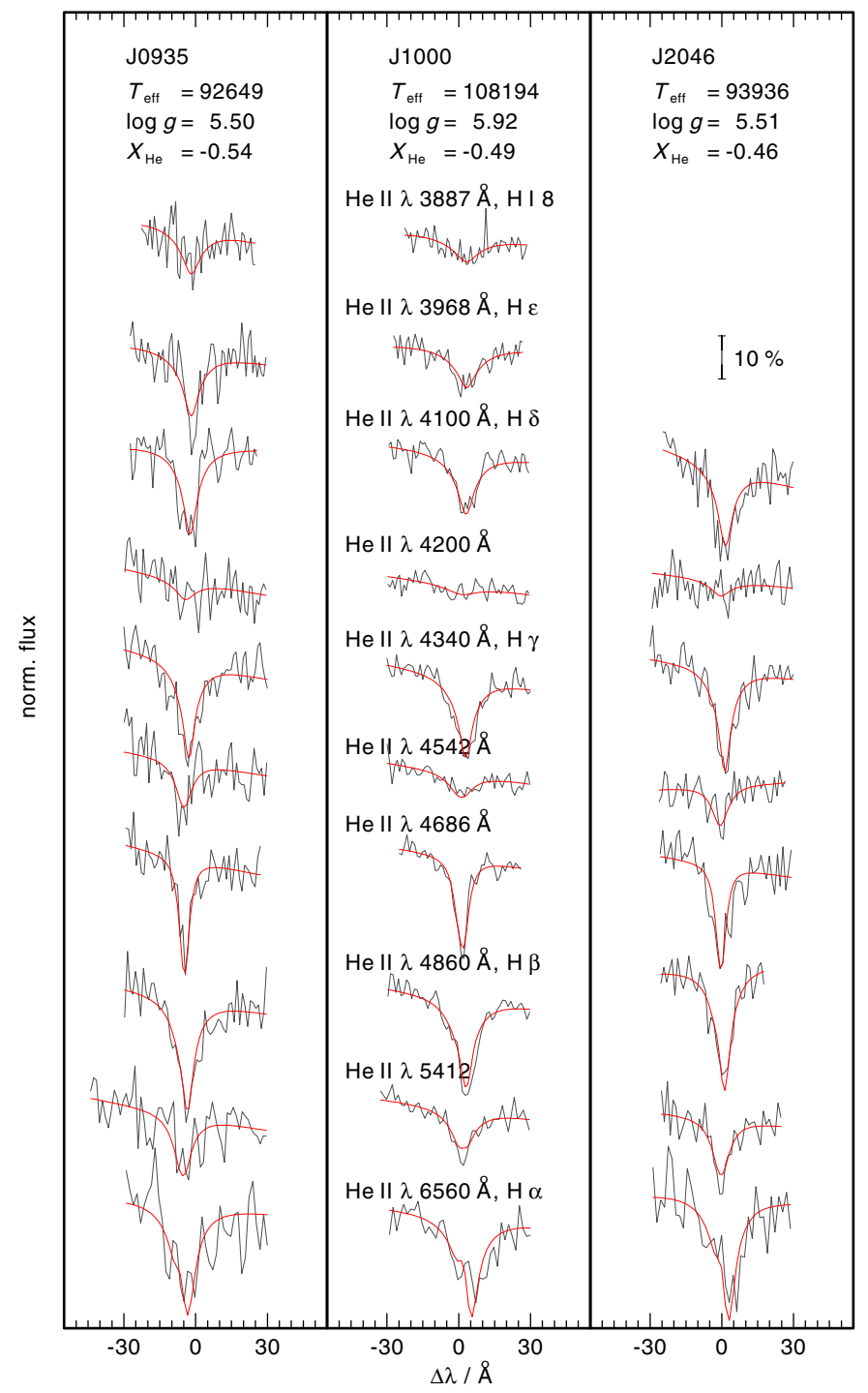

Fig. 2. Balmer and He II lines used to derive $T_{\text {eff }}, \log g$, and the He abundance (given in logarithmic mass fractions) of the three $\mathrm{O}(\mathrm{H})$-type stars. The observations are shown in gray, the best fit models in red. The vertical bar indicates $10 \%$ of the continuum flux.

and 0.6 , by mass). Models above the Eddington limit (i.e., $T_{\text {eff }}>$ $90000 \mathrm{~K}$ for $\log g=4.75, T_{\mathrm{eff}}>100000 \mathrm{~K}$ for $\log g=5.00$, and $T_{\text {eff }}>120000 \mathrm{~K}$ for $\log g=5.25$ ) were not calculated. For He II 20 levels were considered in non-LTE, and for He I 29 levels if $T_{\text {eff }}<100000 \mathrm{~K}$, and five levels if $T_{\text {eff }} \geq 100000 \mathrm{~K}$. For H I 15 levels were considered in non-LTE. The parameter fit was then performed by means of a $\chi^{2}$ minimization technique with SPAS (Spectrum Plotting and Analysing Suite, Hirsch 2009), which is based on the FITSB2 routine (Napiwotzki 1999). We fitted all Balmer and He II lines detected in the SDSS spectra of these stars. He II $\lambda 3968 \AA / \mathrm{H} \epsilon$ and He II $\lambda 3887 \AA / \mathrm{H}$ I $\lambda 3889 \AA$ were not fitted in the case of J2046 owing to the poor quality of the blue part of the spectra. Our best fits are shown in Fig. 2 and the results of our analysis are summarized in Table 1.

The spectra were previously analysed by Geier et al. (2015) using a model grid that was calculated by Stroeer et al. (2007) and we find that they generally underestimated $T_{\text {eff }}$ by 5 to $15 \%$. In addition, our results for J1000 lie outside the grid used by Geier et al. (2015). While the effects of the smaller step size in $T_{\text {eff }}(1250 \mathrm{~K}$ instead of $5000 \mathrm{~K})$ of our grid, are relatively 
Table 1. Stellar parameter.

\begin{tabular}{lllllllll}
\hline \hline \multirow{2}{*}{ Name } & $\begin{array}{l}\text { Spectral } \\
\text { type }\end{array}$ & \multicolumn{1}{l}{$\begin{array}{l}T_{\text {eff }}[\mathrm{K}] \\
\end{array}$} & $\log g$ & $X_{\mathrm{He}}$ & $\begin{array}{l}M \\
{\left[M_{\odot}\right]}\end{array}$ & $\log \left(L / L_{\odot}\right)$ & $\begin{array}{l}d \\
{[\mathrm{kpc}]}\end{array}$ & $\begin{array}{l}z \\
{[\mathrm{kpc}]}\end{array}$ \\
\hline $\mathrm{J} 0935$ & $\mathrm{O}(\mathrm{H})$ & $92649 \pm 11358$ & $5.50 \pm 0.12$ & $-0.46 \pm 0.09$ & $0.54_{-0.01}^{+0.05}$ & $3.5_{-0.2}^{+0.4}$ & $16.7_{-2.2}^{+2.7}$ & $12.1_{-1.6}^{+1.8}$ \\
$\mathrm{~J} 1000$ & $\mathrm{O}(\mathrm{H})$ & $108194 \pm 8356$ & $5.92 \pm 0.09$ & $-0.49 \pm 0.06$ & $0.54_{-0.01}^{+0.02}$ & $3.4_{-0.1}^{+0.2}$ & $8.0_{-0.8}^{+0.9}$ & $5.2_{-0.5}^{+0.6}$ \\
$\mathrm{~J} 2046$ & $\mathrm{O}(\mathrm{H})$ & $93936 \pm 21166$ & $5.51 \pm 0.32$ & $-0.54 \pm 0.16$ & $0.54_{-0.01}^{+0.08}$ & $3.5_{-0.2}^{+0.5}$ & $10.8_{-3.3}^{+4.8}$ & $-5.2_{-2.3}^{+1.6}$ \\
$\mathrm{~J} 1556$ & $\mathrm{PG} 1159$ & $100000_{-10000}^{+15000}$ & $5.30 \pm 0.30$ & $-0.07 \pm 0.03$ & $0.57_{-0.05}^{+0.13}$ & $3.8_{-0.4}^{+0.5}$ & $16.3_{-4.8}^{+6.7}$ & $12.3_{-3.6}^{+5.1}$ \\
$\mathrm{~J} 0757^{a}$ & $\mathrm{O}(\mathrm{He})$ & $80000 \pm 20000$ & $5.00 \pm 0.30$ & $-0.01 \pm 0.01$ & $0.53_{-0.05}^{+0.21 a}$ & $3.7_{-0.3}^{+0.6 b}$ & $26.8_{-7.8}^{+11.1 b}$ & $10.3_{-3.0}^{+4.3 b}$ \\
& & & & & $0.73_{-0.08}^{+0.27 c}$ & $3.9_{-0.5}^{+1.0 c}$ & $31.5_{-9.2}^{+13.0 c}$ & $12.1_{-3.5}^{+5.0 c}$ \\
\hline
\end{tabular}

Notes. He abundances are given in logarithmic mass fractions. ${ }^{(a)}$ Atmospheric parameters taken from Werner et al. (2014). ${ }^{(b)}$ Based on (V)LTP tracks of Miller Bertolami \& Althaus $(2007,2006) .{ }^{(c)}$ Based on double He-white dwarf merger tracks of Zhang \& Jeffery (2012a,b).

small ( $T_{\text {eff }}$ is usually underestimated by $1 \%$ when using a finer grid), the main influence can be attributed, to a large extent, to higher $T_{\text {eff }}(140000 \mathrm{~K}$ instead of $100000 \mathrm{~K})$, as well as in the use of the Stark line-broadening tables provided by Tremblay \& Bergeron (2009), rather than those provided by Lemke (1997).

We note that the errors given in Table 1 correspond to formal fitting errors only. The neglect of metal opacities in the model-atmosphere calculations can lead to significant systematic errors in the inferred atmospheric parameters of very hot stars. Latour et al. $(2010,2015)$ showed that for sdO stars hotter than about $70000 \mathrm{~K}, T_{\text {eff }}$ and $\log g$ are typically underestimated by 5 to $10 \%$ and 0.15 dex, respectively.

However, even taking these effects into consideration, all stars lie clearly in the post-AGB region of the $\log T_{\text {eff }}-\log g$ diagram shortly before they reach their maximum $T_{\text {eff }}$ and then cool down as white dwarfs (Fig. 3). Their He abundances are slightly supersolar (1.2-1.4 × solar, solar abundance according to Asplund et al. 2009). To distinguish these stars from sdO stars, which are usually associated with AGB-manqué stars, which are stars that fail to evolve through the AGB, we employ the spectral classification scheme of Mendez (1991) and classify them as $\mathrm{O}(\mathrm{H})$ type stars.

\subsubsection{H-deficient stars}

J0757 has been analysed by Werner et al. (2014), who derived $T_{\text {eff }}=80000 \mathrm{~K}, \log g=5.00$, and a $\mathrm{C}$ abundance of $C=0.006$ (by mass). Thus, the star belongs to the class of C-rich $\mathrm{O}(\mathrm{He})$ stars.

The $\mathrm{C}$ lines in the spectrum of $\mathrm{J} 1556$ are stronger and more numerous compared to those found in the spectrum of J0757. In Fig. 4 the co-added SDSS spectrum of J1556 is shown. Besides photospheric lines of He II and C IV, we could identify lines of $\mathrm{N} \mathrm{V}$ and $\mathrm{OV}$. Constraints on the effective temperature can already be applied from the absence of He I lines ( $\left.T_{\text {eff }} \geq 70000 \mathrm{~K}\right)$ and the C IV $\lambda \lambda 5801,5812 \AA$ lines, which appear in emission ( $T_{\text {eff }} \geq 90000 \mathrm{~K}$ ). In addition, the fact that the $\mathrm{NV}$ lines at $4604 \AA$ and $4620 \AA$ appear in absorption allows us to put an upper limit of $T_{\text {eff }} \leq 115000 \mathrm{~K}$ (Rauch et al. 1994).

For the spectral analysis of J1556 we firstly calculated a model grid including only the opacities of $\mathrm{H}, \mathrm{He}$, and $\mathrm{C}$. For $\mathrm{H}$ and $\mathrm{He}$, non-LTE levels were used, as described in Sect. 3.1.1. The $\mathrm{C}$ model atom includes the ionization stages III-V and 30, 54, and 1 non-LTE levels were considered for C III, C IV, and C V, respectively. Our model grid spans $T_{\mathrm{eff}}=90000-120000 \mathrm{~K}$ and $\log g=5.0-6.7$ and $\mathrm{C}$ abundances in the range of $0.33-0.01$

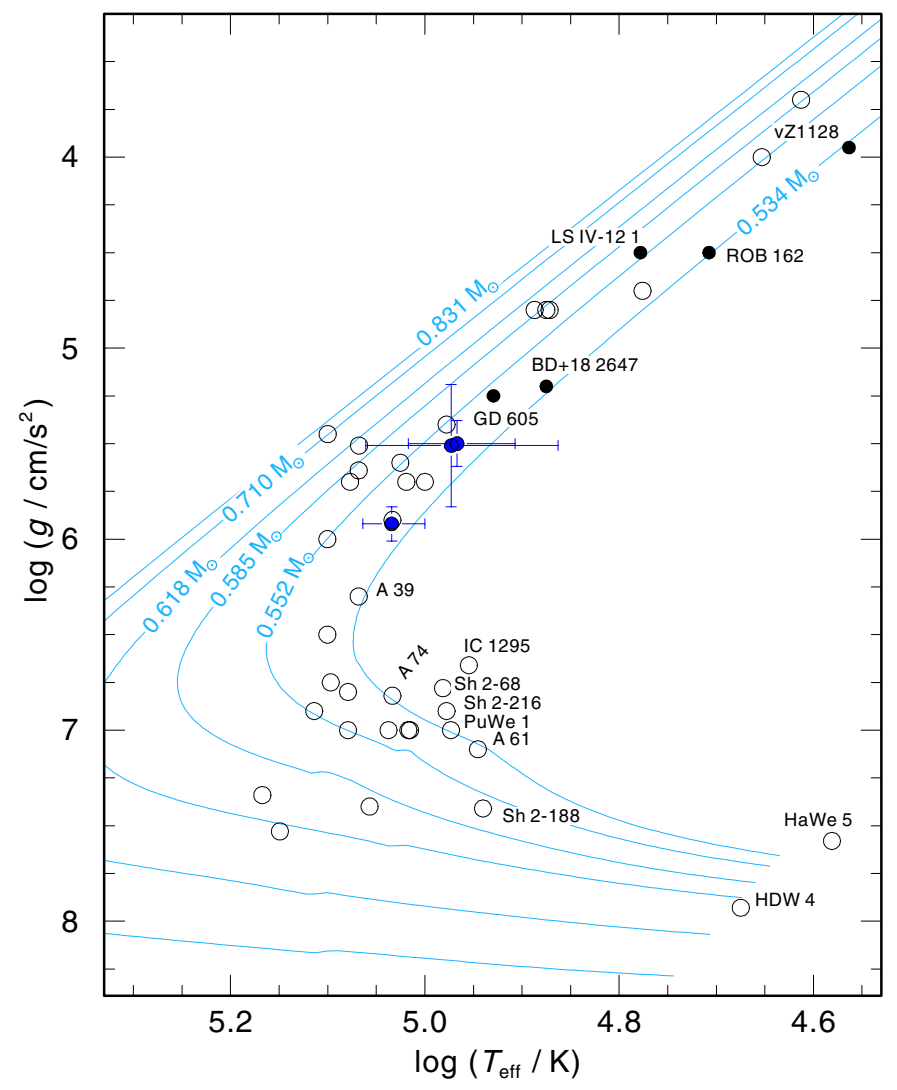

Fig. 3. Locations of our three $\mathrm{O}(\mathrm{H})$ stars (filled blue circles with error bars) are compared with H-burning post-AGB evolutionary tracks (blue lines labeled with stellar masses, Miller Bertolami 2016). Also shown are the locations of naked $\mathrm{O}(\mathrm{H})$-type stars (filled circles, Chayer et al. 2015; Fontaine et al. 2008; Bauer \& Husfeld 1995; Heber \& Hunger 1987; Heber \& Kudritzki 1986), as well as the locations of H-rich CSPNe (open circles, Ziegler 2012; Herald \& Bianchi 2011; Gianninas et al. 2010; Rauch et al. 2007; Napiwotzki 1999).

(by mass) were considered, The grid is, however, not complete owing to the occurrence of numerical instabilities. The fitting of the spectrum was then carried out in an iterative process, in which the parameters of the model have been changed successively until a good agreement with the observation was achieved. The best fit was found for $T_{\text {eff }}=100000 \mathrm{~K}, \log g=5.33$, and $C=0.15$. No traces of $\mathrm{H}$ could be detected. Unfortunately, we did not succeed in calculating numerically stable models that 


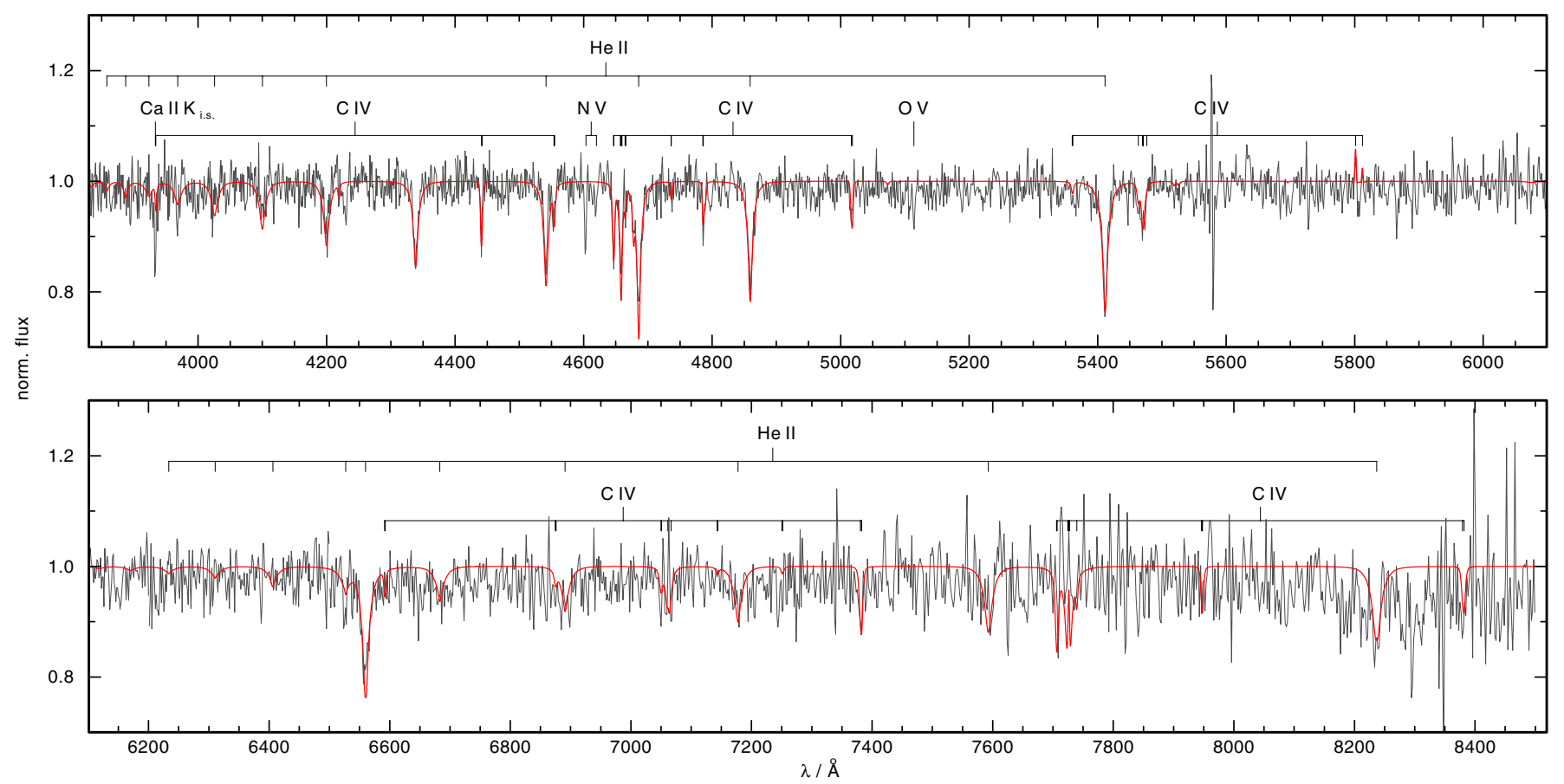

Fig. 4. Coadded and normalized SDSS spectrum (gray) of the PG 1159 star SDSS J155610.40+254640.3. The best fit model (red) including He and $\mathrm{C}$ lines is superimposed and the positions of identified spectral lines are indicated.

also included the opacities of $\mathrm{N}$ and $\mathrm{O}$ at such low surface gravities, which precluded the abundances determination of those elements.

The $\mathrm{C}$ abundance lies well above the limit set by Werner et al. (2014) to distinguish between the PG 1159 stars and the Hedominated DO white dwarfs and $\mathrm{O}(\mathrm{He})$ stars, which only show up to $C=0.03$. Therefore, we classify $\mathrm{J} 155610.40+254640.3$ as a PG 1159 star. It is worth noting, that this is also the PG 1159 star with the lowest surface gravity known so far. Interestingly, its $C$ abundance is rather low compared to the majority of the luminous PG 1159 stars (i.e., they have $\log g<7.0$ ), which typically shows $C=0.5$ (see Fig. 4 in Reindl et al. 2014a for comparison).

\subsection{Masses, luminosities, and distances}

To derive the masses and luminosities of the five stars, we compared their position in the $\log T_{\text {eff }}-\log g$ plane with different evolutionary tracks. Figure 3 shows the location of the three $\mathrm{O}(\mathrm{H})$ stars compared with H-burning post-AGB evolutionary tracks (Miller Bertolami 2016). Because these stars most likely belong to the Galactic halo (see below), we used tracks with $Z=0.001$. We derived $M=0.54 M_{\odot}$ for all $\mathrm{O}(\mathrm{H})$-type stars, which agrees well within the error limits with the mean mass of $0.551 \pm 0.005 M_{\odot}$ of H-rich halo white dwarfs (Kalirai 2012).

In the upper panel of Fig. 5, the locations of the two Hdeficient stars, J0757 and J1556, are compared with a late thermal pulse (LTP) evolutionary track of Miller Bertolami \& Althaus (2007) and very LTP (VLTP) post-AGB tracks of Miller Bertolami \& Althaus (2006). We derived $M=0.57 M_{\odot}$ for $\mathrm{J} 1556$, and $M=0.53 M_{\odot}$ for $\mathrm{J} 0757$. In addition, for $\mathrm{J} 0757$, we derived the mass and luminosity using post-double He white dwarf merger evolutionary tracks of Zhang \& Jeffery (2012a,b) and found $M=0.73 M_{\odot}$ (lower panel of Fig. 5). Within the error limits, J0757 could also be a merger of a CO white dwarf and a He white dwarf, however, there are no post-CO+He white dwarf merger tracks currently available that reach to the region of $\mathrm{O}(\mathrm{He})$ type stars. We emphasise that a reliable spectroscopic mass determination for $\mathrm{J} 0757$ would only be possible if the evolutionary history of this object would be known. Therefore, the parameter listed for J0757 in Table 1, are only valid if a certain scenario is assumed.

Based on the derived masses, we calculated the distances of the stars by using the flux calibration of Heber et al. (1984) for $\lambda_{\text {eff }}=5454 \AA$,

$d[\mathrm{pc}]=7.11 \times 10^{4} \times \sqrt{H_{v} \cdot M \times 10^{0.4 m_{\mathrm{v}_{0}}-\log g}}$,

with $m_{\mathrm{V}_{\mathrm{o}}}=m_{\mathrm{V}}-2.175 c, c=1.47 E_{B-V}$, and the Eddington flux $H_{v}$ at $\lambda_{\text {eff }}$ of the best-fit model atmospheres of each star. Values for $E_{B-V}$ were obtained from Galactic dust extinction maps from Schlafly \& Finkbeiner (2011) and values for $m_{\mathrm{V}}$ were taken from Geier et al. (2015). The distances range from 8.0 to $31.5 \mathrm{kpc}$ and deviate slightly from the values listed in Geier et al. (2015). This is because they used different model fluxes and assumed a canonical mass of $0.6 M_{\odot}$ for all post-AGB stars for their distance determination. We found that all five stars are located far above or below the Galactic plane (Table 1). Since the thick disk only dominates in the region $1 \mathrm{kpc} \lesssim z \lesssim 4 \mathrm{kpc}$ (Kordopatis et al. 2011), we conclude that all stars from our sample probably belong to the Galactic halo.

\section{Search for nebular lines and infrared excess}

All of our five stars are located in the region of CSPNe in the log $T_{\text {eff }}-\log g$ diagram, which suggests that they might be central stars as well. The large distances of the stars, however, imply rather small angular diameters of approximately one arcsec or even less (assuming a typical expansion velocity of $20 \mathrm{~km} \mathrm{~s}^{-1}$ 


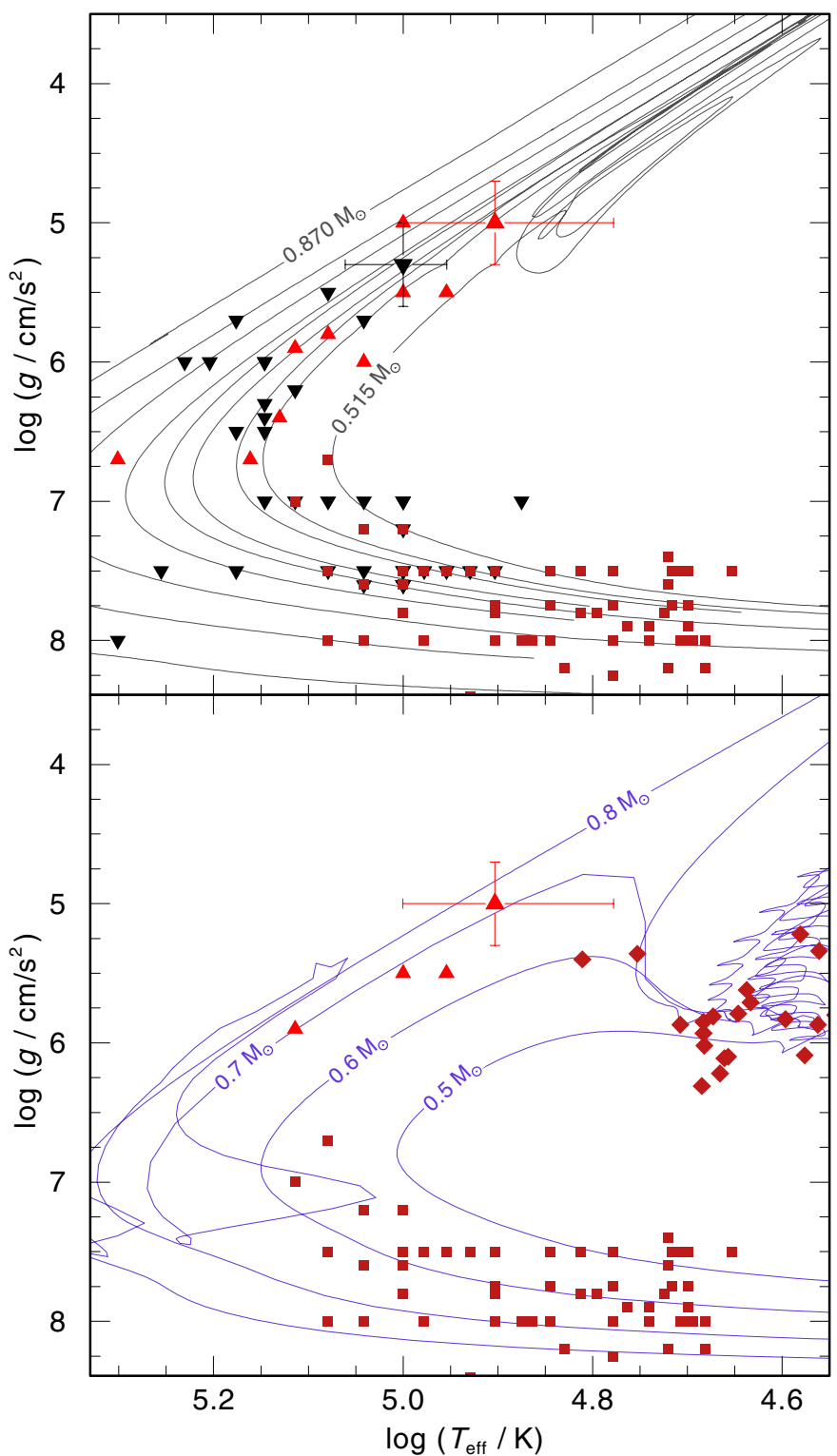

Fig. 5. Locations of the $\mathrm{O}(\mathrm{He})$ star $\mathrm{J} 0757$ (red triangle with error bars) and the PG 1159 star J1556 (black inverted triangle with error bars) and other PG 1159 and $\mathrm{O}(\mathrm{He})$ stars (black inverted triangles and red triangles, respectively, De Marco et al. 2015; Reindl et al. 2014b; Werner \& Rauch 2014; Werner et al. 2014; Gianninas et al. 2010; Wassermann et al. 2010; Werner \& Herwig 2006) in the $T_{\text {eff }}-\log g$ diagram. Their likely successors, the H-deficient white dwarfs (Reindl et al. 2014a; Werner et al. 2014; Mahsereci 2011; Hügelmeyer et al. 2006; Dreizler $\&$ Werner 1996) are indicated by filled-in red squares. The upper panel compares the location of these stars to (V)LTP evolutionary tracks (gray lines) of Miller Bertolami \& Althaus (2007, 2006). For clarity, only two tracks are labeled with stellar masses. Intermediate tracks correspond to $0.530,0.542,0.565,0.584,0.609,0.664,0.741 M_{\odot}$. The lower panel compares only the locations of the $\mathrm{C}$-rich $\mathrm{O}(\mathrm{He})$ stars (red triangles) and $\mathrm{C}$-strong lined $\mathrm{He}$-sdO stars (red rhombs) from the sample of Geier et al. (2015) with post-double He white dwarf merger tracks (purple lines) of Zhang \& Jeffery (2012a,b).

and distances and post-AGB times from Table 3). Thus, it is unlikely a PNe around these stars could be detected via groundbased imaging. Therefore, the search for prominent nebular emission lines is much more promising in the spectra of these stars, i.e., the [O III] $\lambda \lambda$ 4959, $5007 \AA$ emission lines, which are typically the strongest lines for a medium- to high-excitation

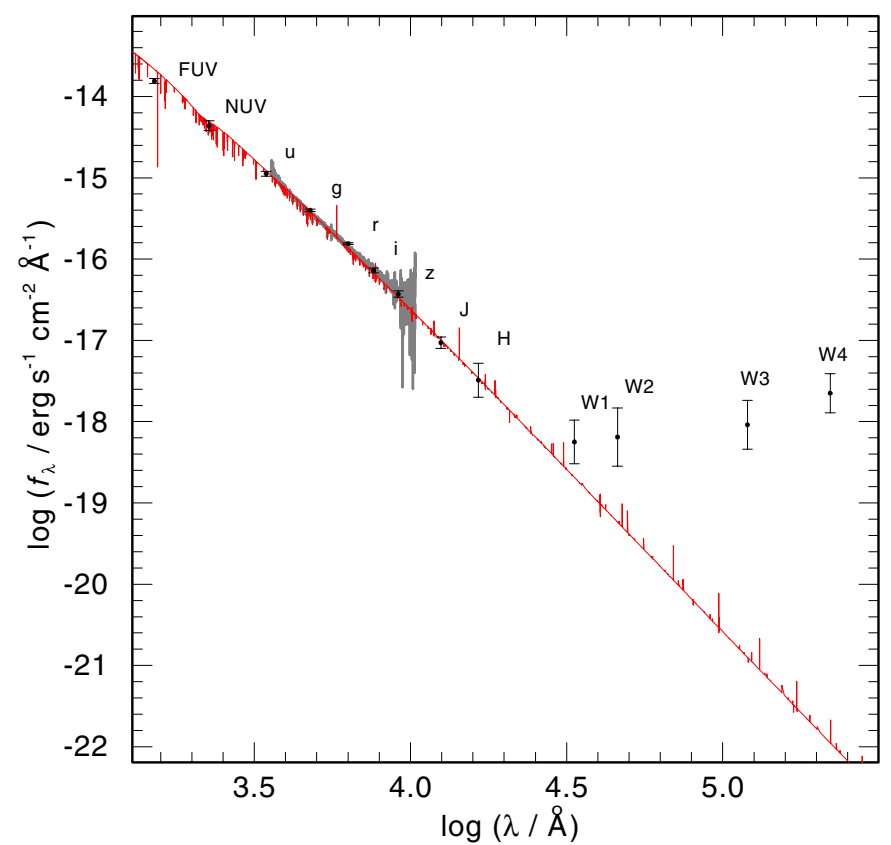

Fig. 6. Colors (black dots with $3 \sigma$ error bars) and the SDSS spectrum (gray) of the PG 1159 star J1556 (gray), compared with a reddened TMAP model SED (red). The emission lines are of photospheric origin. Note that the mismatch of the reddened model flux and the GALEX FUV measurement is due to missing metal opacities in our model atmosphere.

PN. Because of the long exposure times and the much better background subtraction that can be achieved in slit or fibre spectroscopy compared to narrow-band imaging or slitless objective prism imaging spectroscopy, SDSS spectra allow the detection of extremely faint PN (down to 29-30 mag $\operatorname{arcsec}^{-1}$, Yuan \& Liu 2013). However, we could not detect any hint of nebular lines in the spectra of our five stars.

The detection of an infrared (IR) excess can put constraints on the nature of a possible companion, or the presence of a circumstellar disk. To search for possible IR excess emission around our five stars, we cross-matched their positions with the UKIDSS (UKIRT Infrared Deep Sky Survey) DR9 cata$\log$ (Lawrence et al. 2013) and the WISE (Wide-field Infrared Survey Explorer) catalog (Cutri \& et al. 2014). We applied a search radius of 2 arcsec. For the $\mathrm{O}(\mathrm{H})$ stars J0935 and J2046, no IR colors were found, but for J1000 and the PG 1159 star J1556, we found $J$ and $H$ entries. For the $\mathrm{O}(\mathrm{He})$ star $\mathrm{J} 0757$, we found a $J$ value. The PG 1159 star J1556 is the only object with entries in the WISE catalog. In addition, GALEX FUV and NUV (Bianchi et al. 2014) and SDSS magnitudes (Ahn et al. 2012) were obtained for all objects. Magnitudes and reddening of the five stars are summarized in Table 2. SDSS, UKIDSS, and WISE magnitudes were converted into fluxes as outlined in Verbeek et al. (2014). For the GALEX magnitudes conversions ${ }^{3}$, we used

$$
\begin{aligned}
& f_{F U V}\left[\operatorname{erg~s}^{-1} \mathrm{~cm}^{-2} \AA^{-1}\right]=1.40 \times 10^{-15} 10^{-0.4(F U V-18.82)} \\
& f_{N U V}\left[\operatorname{erg~s}^{-1} \mathrm{~cm}^{-2} \AA^{-1}\right]=2.06 \times 10^{-16} 10^{-0.4(N U V-20.08)} .
\end{aligned}
$$

Then, we corrected the TMAP model flux of our best fit for each star for interstellar reddening, using the reddening law of

\footnotetext{
3 http://asd.gsfc.nasa.gov/archive/galex/FAQ/ counts_background.html
} 
Table 2. Magnitudes and reddening of the stars.

\begin{tabular}{lrrrrr}
\hline \hline Object & \multicolumn{1}{c}{ J0935 } & \multicolumn{1}{c}{$\mathrm{J} 1000$} & \multicolumn{1}{c}{$\mathrm{J} 2046$} & \multicolumn{1}{c}{$\mathrm{J} 1556$} & \multicolumn{1}{c}{ J0757 } \\
\hline$E_{B-V}$ & $0.018 \pm 0.001$ & $0.036 \pm 0.002$ & $0.058 \pm 0.003$ & $0.058 \pm 0.005$ & $0.030 \pm 0.001$ \\
$F U V$ & $16.325 \pm 0.019$ & $15.799 \pm 0.019$ & $15.993 \pm 0.034$ & $16.157 \pm 0.036$ & $16.755 \pm 0.037$ \\
$N U V$ & $16.899 \pm 0.015$ & $16.431 \pm 0.016$ & $16.555 \pm 0.031$ & $16.712 \pm 0.018$ & $17.297 \pm 0.033$ \\
$u$ & $17.672 \pm 0.010$ & $17.053 \pm 0.007$ & $17.075 \pm 0.008$ & $17.241 \pm 0.009$ & $17.837 \pm 0.012$ \\
$g$ & $18.114 \pm 0.006$ & $17.483 \pm 0.005$ & $17.450 \pm 0.005$ & $17.675 \pm 0.006$ & $18.280 \pm 0.007$ \\
$r$ & $18.681 \pm 0.009$ & $18.019 \pm 0.007$ & $17.884 \pm 0.007$ & $18.141 \pm 0.007$ & $18.775 \pm 0.010$ \\
$i$ & $19.075 \pm 0.014$ & $18.372 \pm 0.009$ & $18.207 \pm 0.009$ & $18.518 \pm 0.010$ & $19.166 \pm 0.015$ \\
$z$ & $19.413 \pm 0.051$ & $18.747 \pm 0.034$ & $18.505 \pm 0.035$ & $18.860 \pm 0.034$ & $19.477 \pm 0.058$ \\
$J$ & & $18.511 \pm 0.052$ & & $18.882 \pm 0.178$ & $19.217 \pm 0.117$ \\
$H$ & & $19.113 \pm 0.198$ & & $18.746 \pm 0.099$ & \\
$W 1$ & & & & $17.305 \pm 0.130$ & \\
$W 2$ & & & & $16.282 \pm 0.192$ & \\
$W 3$ & & & & $8.250 \pm 0.190$ & \\
$W 4$ & & & & & \\
\hline
\end{tabular}

Fitzpatrick (1999). Again, we used the values for $E_{B-V}$ from the Galactic dust extinction maps of Schlafly \& Finkbeiner (2011). Thereafter, we normalized the reddened model flux to the SDSS $g$ magnitude and checked if the other color measurements are within $3 \sigma$ in agreement with the model fluxes.

A perfect agreement with the color measurements and the reddened model flux was found for the three $\mathrm{O}(\mathrm{H})$ stars and the $\mathrm{O}(\mathrm{He})$ star, i.e., no evidence for an excess was found in the near IR. The PG 1159 star J1556, however, displays a clear excess in all WISE colors (Fig. 6). Because of the lack of WISE measurements, a search for a far-IR excess was not possible for the four other stars. In the (W2-W3), (W3-W4) diagram, the WISE colors of J1556 agree with the bulk of PNe (Kronberger et al. 2014). However, as mentioned above, no nebular emission lines are visible in the SDSS spectrum (total exposure time $\approx 2.25 \mathrm{~h}$ ).

An IR excess that shows up strongly in the Spitzer IRAC bands (Xu \& Jura 2012), was found around some warm (i.e., $T_{\text {eff }} \leq 25000 \mathrm{~K}$ ) white dwarfs. This IR excess is interpreted as originating from a small hot disk, which is due to the infall and destruction of single asteroids that come within the star's Roche limit (Gänsicke et al. 2006). However, we exclude the presence of a debris disk in the case of J1556, because at such high $T_{\text {eff }}$, solids would be sublimated into gaseous disks (von Hippel et al. 2007). In addition the double-peaked Ca II triplet, which is the hallmark of a gaseous, rotating disk (Young et al. 1981; Horne \& Marsh 1986), cannot be detected.

Cold dust disks, which are located much farther from the star ( $\approx 50$ au compared to $\leq 0.01$ au for the debris disks) have been announced for a number of hot white dwarfs and CSPNe and are likely formed by mass loss from the stars during their AGB phase (Clayton et al. 2014; Chu et al. 2011). These disks, however, were found to be relatively cool $(\approx 100 \mathrm{~K}$ compared $\approx 1000 \mathrm{~K}$ for the debris disks) and should show up only in the $W 4$ band. The relatively flat IR excess in J1556 cannot be fitted with a single blackbody and looks very similar to the case of the CSPN K 1-22. Chu et al. (2011) showed that for K 1-22, the IR excess is a superposition of the photospheric emission of the CSPN and a red companion as well as the dust continuum. Thus, it is likely that more than one source contributes to the IR excess of $\mathrm{J} 1556$ as well.
Finally, we note that the IR excess could also have an extragalactic origin. We corrected the WISE colors of J1556 for the stellar flux contribution and obtained $W 1=18.40, W 2=16.54$, $W 3=12.23$, and $W 4=8.93$. This leads to $W 1-W 2=1.86$ and $W 2-W 3=4.31$, which corresponds to the region of luminous IR galaxies or Sbc star-burst galaxies in the (W1-W2), (W2W3) diagram (Fig. 12 in Wright et al. 2010). The WISE-excess might therefore be caused by a chance alignment of such a background galaxy with J1556. However, the probability for such an alignment of two very peculiar classes of objects seems to be very unlikely.

\section{Discussion}

The high RV variations - partly on short timescales - detected in four of our stars are a strong hint that they are part of close binary systems. Unlike hot subdwarfs, which are expected to have already lost almost their entire envelope on the RGB via stable Roche lobe overflow and/or common envelope evolution, the mass loss on the RGB of post-AGB close binaries must have been weaker. This is because only stars with a sufficiently thick envelope are able to maintain $\mathrm{H}$-shell burning and to ascend the AGB.

The post-AGB phase (i.e., the evolution from the AGB towards the maximum $T_{\text {eff }}$ ) is very short-lived. The evolutionary timescales are very dependent on the stellar mass, so that a $0.53 M_{\odot}$ star needs about $80000 \mathrm{yr}$ to reach its maximum $T_{\text {eff }}$, while a $0.83 M_{\odot}$ star reaches its maximum $T_{\text {eff }}$ within less than $1000 \mathrm{yr}$ (Miller Bertolami 2016). Every post-AGB star is expected to eventually become a CSPN, if the star is able to ionize the previously ejected outer layers before the expanding envelope becomes too tenuous to appear as a PN.

As mentioned above, all of our five stars are located in the $\log T_{\text {eff }}-\log g$ diagram just amongst the CSPNe. However, no nebular lines can be detected in their spectra. Therefore in the following, we discuss the evolutionary status of these objects, taking the absence of a PN around these stars into account, as well as their possible close binary nature. 


\subsection{The PG 1159 star J1556}

The phenomenon of the "missing" PN is well known for the H-deficient PG 1159 and $\mathrm{O}(\mathrm{He})$ stars. Only 15 of the 47 currently known PG 1159 stars are confirmed CSPNe, which can be understood with reference to their evolutionary history. PG 1159 stars are believed to be the result of a (V)LTP that occurs either during the blueward excursion of the post-AGB star (LTP), or during its early white dwarf cooling phase (VLTP). The release of nuclear energy by the flashing helium shell forces the already very compact star to expand back to giant dimensions - the socalled born-again scenario (Fujimoto 1977; Schönberner 1979; Iben et al. 1983; Althaus et al. 2005). The absence of hydrogen in the atmosphere of J1556, combined with the appearance of $\mathrm{NV}$ lines in the SDSS spectrum, suggests a VLTP (Werner \& Herwig 2006). Since a three times longer evolutionary timescale is predicted for He-burners (Iben et al. 1983) and, given that J1556 is going through the post-AGB phase for the second time, the missing PN can be understood very well.

Should the RV variations in $\mathrm{J} 1556$ be confirmed as originating from a close companion, then J1556 would be just the second known PG 1159 close binary system besides SDSS J212531.92-010745.9 (Nagel et al. 2006; Schuh et al. 2009; Shimansky et al. 2015). The companion in the latter system is a low-mass main-sequence star as it is obvious from the reflection effect in the light curve and from the hydrogen Balmer series, which appears in emission owing to the radiation of the PG 1159 star (Nagel et al. 2006). However, in the spectra of J1556, we cannot find evidence of these type of emission lines. The IR excess of J1556 shows up only in the WISE bands, which suggest that a possible companion to J1556 might be even less massive. We emphasise that SDSS J212531.92-010745.9 is at least two orders of magnitude less luminous when compared to $\mathrm{J} 1556$, and thus it is much more difficult to detect a low-mass main-sequence star for the latter.

Amongst the H-deficient Wolf Rayet type central stars, which are believed to be the progenitors of the PG 1159 stars (Werner \& Herwig 2006), only three close binary systems have been discovered so far (Hajduk et al. 2010; Manick et al. 2015). This leads to a known close binary fraction of about $5 \%$ amongst these C-dominated objects ${ }^{4}$, which is significantly less compared to the overall close binary fraction of CSPNe $(\approx 15-20 \%$, Bond 2000; Miszalski et al. 2009). This suggests, that binary interaction may not play a fundamental role in forming $C$-dominated stars. Definitive statements can, however, only be made after a systematic search of close binaries amongst these stars.

\subsection{The $\mathrm{O}(\mathrm{He})$ star $\mathrm{J} 0757$}

$\mathrm{J} 0757$ is the first RV variable $\mathrm{O}(\mathrm{He})$ star ever discovered. De Marco et al. (2015) recently discovered the O(He)-type CSPN of Pa 5 (PNG076.3+14.1) as having a consistent photometric period of $1.12 \mathrm{~d}$ in twelve individual Kepler quarters. However, they failed to detect RV shifts larger than $5 \mathrm{~km} \mathrm{~s}^{-1}$. They argue that the photometric variability may be explained by the influence of an orbiting planet or magnetic activity, but is most likely due to an evolved companion in a nearly-pole-on orbit. Given that there are currently only ten $\mathrm{O}(\mathrm{He})$ stars known, the confirmation of close binaries to J0757 and Pa 5 would already indicate a binary fraction of $20 \%$. This may suggest, that

\footnotetext{
4 There are currently about 60 Wolf Rayet type central stars known (H. Todt, priv. comm.).
}

close binary evolution is, at least for some of the He-dominated objects, an important formation channel.

Also among He-dominated stars, a PN seems to be the exception rather than the rule. In addition, it appears that the presence of a $\mathrm{PN}$ is restricted to $\mathrm{N}$-rich $\mathrm{O}(\mathrm{He})$ stars and [WN]-type CSPNe only, which suggests that these objects are formed differently to $\mathrm{C}$-rich or $\mathrm{C}+\mathrm{N}$-rich He-dominated objects (Reindl et al. 2014b). The possibility that the latter two subclasses are formed via the merger of two white dwarfs naturally explains the missing PN, because of the very long predicted post-merger times of the stars. However, the confirmation of a close companion to J0757 would question the merger scenario as triple stars systems are arranged hierarchically. Moreover, it is questionable whether it is possible for unstable mass transfer to stop before the merger of two white dwarfs is complete and, thus, leaving behind a very low-mass close companion (Justham et al. 2010; Han \& Webbink 1999).

We consider therefore yet another possibility to explain both, the missing PN as well as the extreme maximum RV shift of $107.0 \pm 22.0 \mathrm{~km} \mathrm{~s}^{-1}$ within only $31 \mathrm{~min}$ (which is already pointing toward a very close binary system), namely that J0757 could have evolved from an AMCVn type system which stopped accreting. AMCVn stars are He-transferring ultracompact binary systems with an orbital period below $1 \mathrm{~h}$. The mass ratio of these systems is expected to be sufficently low and, therefore, stable mass transfer occurs. These systems prevent the merger and, with decreasing accretion rates, they evolve towards longer orbital periods (Yungelson et al. 2002; Marsh et al. 2004; Nelemans et al. 2010). The accretors of these sytems may experience stable He-burning (Piersanti et al. 2014, 2015). In the case of the progenitor of J0757 being a He WD, which ignited He-burning, the post accretion evolution might look similar to that of the post-double He white dwarf merger models of Zhang \& Jeffery (2012a,b) and would thus imply that J0757 must have evolved through the region of the central He burning He-sdO stars (lower panel of Fig. 5).

Interestingly, we find that the great majority (up to 84\%) of the RV-variable He-sdO stars discovered by Geier et al. (2015), belong to the class of C-rich He-dominated objects ${ }^{5}$, just like $\mathrm{J} 0757$. We also note that the irregular RV variations detected by Geier et al. (2015) in some of the He-sdO stars (e.g., the C-rich He-sdO SDSS J232757.46+483755.2), might even support this scenario, if we assume that they are caused by a still present accretion stream (Schwarz et al. 2010).

\subsection{H-rich stars}

$\mathrm{J} 1000$ and J0935 are the first RV variable naked $\mathrm{O}(\mathrm{H})$ stars ever discovered. So far, only five other naked $\mathrm{O}(\mathrm{H})$ stars are known: BD+18 2647 (Bauer \& Husfeld 1995), ROB 162 in the globular cluster NGC 6397 (Heber \& Kudritzki 1986), vZ1128 in the globular cluster M 3 (Chayer et al. 2015), GD 605, which is also considered to be a halo star (Fontaine et al. 2008), as well as the metal-poor $\mathrm{O}(\mathrm{H})$ star LS IV-12 1 (Heber \& Hunger 1987). The fact that no PN is present around these stars is rather unexpected, since in our canonical understanding each star that evolves through the AGB should produce a PN. A simple explanation for their missing PNe could be that their post-AGB times are longer than the time it took for the PNe to disappear

\footnotetext{
Classification based on the presence of $\mathrm{C}$ lines, but absence of $\mathrm{N}$ lines in the SDSS spectra. Either the remaining RV variable He-sdO stars do not show any metal lines $(8 \%)$ or belong to the group of $\mathrm{C}+\mathrm{N}$-rich $\mathrm{He}-$ sdO stars $(8-12 \%)$.
} 
Table 3. Post-AGB times, as derived from H-burning post-AGB tracks of Miller Bertolami (2016) of the eight naked $\mathrm{O}(\mathrm{H})$ stars and some CSPNe with very long post-AGB times.

\begin{tabular}{|c|c|c|}
\hline & Name & $t_{\text {post-AGB }}$ \\
\hline & LS IV-12 $1^{a}$ & $9 \mathrm{kyr}$ \\
\hline & GD $605^{b}$ & $20 \mathrm{kyr}$ \\
\hline & $\mathrm{J} 2046^{c}$ & $31 \mathrm{kyr}$ \\
\hline & $\mathrm{J} 0935^{c}$ & $31 \mathrm{kyr}$ \\
\hline & $\mathrm{J} 1000^{c}$ & $33 \mathrm{kyr}$ \\
\hline & $\mathrm{vZ} 1128^{d}$ & $38 \mathrm{kyr}$ \\
\hline & $\operatorname{ROB} 162^{e}$ & $49 \mathrm{kyr}$ \\
\hline & $\mathrm{BD}+182647^{f}$ & $56 \mathrm{kyr}$ \\
\hline PNG & name & $t_{\text {post-AGB }}$ \\
\hline $047.0+42.4$ & A $39^{g}$ & $73 \mathrm{kyr}$ \\
\hline $072.7-17.1$ & A $74^{h}$ & $80 \mathrm{kyr}$ \\
\hline $158.9+17.8$ & PuWe $1^{h}$ & $80 \mathrm{kyr}$ \\
\hline $025.4-04.7$ & IC $1295^{h}$ & $>80 \mathrm{kyr}$ \\
\hline $030.6+06.2$ & Sh $2-68^{h}$ & $>80 \mathrm{kyr}$ \\
\hline $158.5+00.7$ & Sh $2-216^{i}$ & $>80 \mathrm{kyr}$ \\
\hline $077.6+14.7$ & $\mathrm{~A} 61^{g}$ & $90 \mathrm{kyr}$ \\
\hline $128.0-04.1$ & Sh $2-188^{j}$ & $120 \mathrm{kyr}$ \\
\hline $047.0+42.4$ & A $39^{f}$ & $73 \mathrm{kyr}$ \\
\hline $072.7-17.1$ & $\mathrm{~A} 74^{g}$ & $80 \mathrm{kyr}$ \\
\hline $158.9+17.8$ & PuWe $1^{f}$ & $80 \mathrm{kyr}$ \\
\hline $025.4-04.7$ & IC $1295^{g}$ & $>80 \mathrm{kyr}$ \\
\hline $030.6+06.2$ & Sh $2-68^{g}$ & $>80 \mathrm{kyr}$ \\
\hline $158.5+00.7$ & $\operatorname{Sh} 2-216^{h}$ & $>80 \mathrm{kyr}$ \\
\hline $077.6+14.7$ & $\mathrm{~A} 61^{f}$ & $90 \mathrm{kyr}$ \\
\hline $128.0-04.1$ & $\operatorname{Sh} 2-188^{j}$ & $120 \mathrm{kyr}$ \\
\hline
\end{tabular}

Notes. For objects that are not covered by these tracks, we give lower limits. Post-AGB times derived from atmospheric parameters taken from ${ }^{(a)}$ Heber \& Hunger (1987); ${ }^{(b)}$ Fontaine et al. (2008); ${ }^{(c)}$ this work; ${ }^{(d)}$ Chayer et al. (2015); ${ }^{(e)}$ Heber \& Kudritzki (1986); ${ }^{(f)}$ Bauer \& Husfeld (1995); ${ }^{(g)}$ Napiwotzki (1999); ${ }^{(h)}$ Ziegler (2012); ${ }^{(i)}$ Rauch et al. (2007); ${ }^{(j)}$ Gianninas et al. (2010).

into the interstellar medium. This possibility is, however, put into doubt when we look in Fig. 3, where we show the locations of H-rich CSPNe found in the literature in addition to the eight known naked $\mathrm{O}(\mathrm{H})$ stars. We derived the post-AGB times from the post-AGB tracks of Miller Bertolami (2016) for the seven naked $\mathrm{O}(\mathrm{H})$ stars, as well as the H-rich CSPNe. As can be seen from Table 3, we found that - compared to the eight naked $\mathrm{O}(\mathrm{H})$ stars - there are several CSPNe with apparently much longer post-AGB times ${ }^{6}$.

On that basis, it is not clear why these stars still harbor a PNe, while the eight naked $\mathrm{O}(\mathrm{H})$ stars do not, and it raises the question: Does every post-AGB star, and in particular every close binary post-AGB system, necessarily produce a PNe? This is particularly important to understand, given the proposed role of close binary post-AGB stars in the formation of asymmetrical PNe. We emphasise that, if J1000 and J0935 indeed turn out to be close binary systems, then this would lead to a close binary

\footnotetext{
6 Note that for the two DA white dwarf CSPNe HaWe 5 and HDW 4, Napiwotzki (1999) even found post-AGB times of several million years, which, however, strongly contradicted the very short kinematical ages of both PNe (less than 4 kyr). Napiwotzki (1999) suggested that the PNe of these stars might actually be ejected nova shells.
}

fraction of $25 \%$ amongst naked $\mathrm{O}(\mathrm{H})$ stars, which is even higher than that found for the CSPNe.

An alternative solution to the non-ejection of a PNe of apparently naked $\mathrm{O}(\mathrm{H})$ stars was suggested by Heber \& Hunger (1987), namely that these stars also suffered an LTP. Contrary to the VLTP scenario, which always results in a H-deficient surface composition, since $\mathrm{H}$ is mixed and already burnt during the thermal pulse, the LTP does not necessarily predict an $\mathrm{H}$-poor star. This is because, in the case of an LTP, the convective shell triggered by excessive He burning is not able to penetrate the H-rich envelope from below because the entropy jump across the $\mathrm{He} / \mathrm{H}$ interface is too large. Only when the star evolves back to its Hayashi limit on the AGB ( $\left.T_{\text {eff }} \lesssim 7000 \mathrm{~K}\right)$, envelope convection sets in again (Blöcker \& Schönberner 1996, 1997; Schönberner 2008). Evolutionary calculations without overshoot (e.g., Blöcker \& Schönberner 1997) predict only mild, if any, mixing. The envelope convection does not reach the layers enriched with carbon, and no third dredge-up occurs. Later calculations by Herwig (2001) show that, if overshoot is applied to all convective regions, AGB models show efficient dredge-up, even for very low envelope masses and thus produce $\mathrm{H}$-deficient stars. Observational proof for the validity of the latter case is offered by FG Sge, the only star known to date that certainly must have suffered an LTP (Schönberner 2008). This star was actually observed evolving back to the AGB where the $\mathrm{H}$ fraction in its atmosphere got diluted significantly (from 0.9 to 0.01 , by number, Jeffery \& Schönberner 2006).

Finally, it is interesting to note that there are also some F or $G$ type supergiants which have already departed from the AGB but lack a reflection nebula and, as such, are not expected to evolve into a CSPN. These much cooler objects (compared to the $\mathrm{O}(\mathrm{H})$ stars discussed above) show the presence of a compact disk with an inner radius of $\approx 15$ AU (Deroo et al. 2006, 2007) and, in addition, they were found to reside in binary systems. They have orbital periods between a hundred and a couple of thousands days, and probably unevolved (very) low initial mass companions (van Winckel et al. 2009). It is thought, that these stars probably had a pre-PN before, but that the re-accretion of material has stalled the blueward evolution of the post-AGB star, which therefore provided the circumstellar material enough time to disperse before it could became ionized (De Marco 2014).

\section{Conclusions}

Our non-LTE model atmosphere analysis revealed that all five stars, which were recently discovered by the MUCHFUSS project as RV stars, lie in the post-AGB region of the HRD. The RV variations were not significant in the case of the $\mathrm{O}(\mathrm{H})$ star J2046, but in the cases of the other two $\mathrm{O}(\mathrm{H})$ stars (J1000, J0935), the O(He) star (J0757), and the PG 1159 star (J1556). This reveals $\mathrm{J} 0757$ as the first RV variable $\mathrm{O}(\mathrm{He})$ star ever discovered, J1556 as the only second known RV variable PG 1159 star, and J1000 and J0935 as the first RV variable $\mathrm{O}(\mathrm{H})$ stars that do not show any hint of a PN.

The absence of a PN around the PG 1159 star J1556 can be explained well in terms of a VLTP evolution, which can also accounts for the high $\mathrm{C}$ abundance. Given the currently known low binary fraction of C-dominated stars, at the moment it appears that binary interactions do not play a crucial role in the formation of these stars.

In the case of the $\mathrm{O}(\mathrm{He})$ star $\mathrm{J} 0757$, we speculate that it was once part of a compact He transferring binary systems in which the mass transfer had stopped after a certain time, leaving behind 
a low-mass close companion. As a result, we could explain both the absence of a PN as well as the RV shift of $107.0 \pm 22.0 \mathrm{~km} \mathrm{~s}^{-1}$ measured as within only $31 \mathrm{~min}$. These speculations definitely need more observational, as well as theoretical, support, however, they might offer an as yet unconsidered evolutionary channel for the mysterious He-dominated objects.

Various explanations could explain the missing $\mathrm{PNe}$ around the $\mathrm{O}(\mathrm{H})$-type stars. The possibility that the post-AGB times of the naked $\mathrm{O}(\mathrm{H})$ stars are longer than it took for the PNe to disappear into the interstellar medium seems odd when there are several CSPNe with much longer post-AGB times. Besides the non-ejection of a PN, the occurrence of a LTP or the re-accretion of the PN in the previous post-AGB evolution, offer possible explanations for stars not harbouring a PN (anymore).

The search for an IR excess was successful only in the case of J1556, which displays a clear IR excess in all WISE colors. To understand its nature, spectroscopic follow-up observations are necessary. We should, however, bear in mind that it is very difficult to detect a close companion via the IR excess method given the very high luminosities of stars in the post-AGB region (our stars have several thousand times the solar luminosity, Table 1). Thus, the presence of a late-type companion to the other stars in our sample cannot be ruled out from the outset.

Acknowledgements. N.R. was supported by DFG (grant WE 1312/41-1) and DLR (grant 50 OR 1507). We thank Marcelo Miguel Miller Bertolami for providing us with the post-AGB tracks before they were published. We thank Thomas Rauch and Peter van Hoof for helpful discussions and comments. Based on observations collected at the Centro Astronómico Hispano Alemán (CAHA) at Calar Alto, operated jointly by the Max-Planck Institut für Astronomie and the Instituto de Astrofísica de Andalucía (CSIC). Based on observations with the William Herschel and Isaac Newton Telescopes operated by the Isaac Newton Group at the Observatorio del Roque de los Muchachos of the Instituto de Astrofisica de Canarias on the island of La Palma, Spain. Funding for SDSSIII has been provided by the Alfred P. Sloan Foundation, the Participating Institutions, the National Science Foundation, and the US Department of Energy Office of Science. The SDSS-III web site is http://www . sdss3.org/. SDSSIII is managed by the Astrophysical Research Consortium for the Participating Institutions of the SDSS-III Collaboration including the University of Arizona, the Brazilian Participation Group, Brookhaven National Laboratory, Carnegie Mellon University, University of Florida, the French Participation Group, the German Participation Group, Harvard University, the Instituto de Astrofisica de Canarias, the Michigan State/Notre Dame/JINA Participation Group, Johns Hopkins University, Lawrence Berkeley National Laboratory, Max Planck Institute for Astrophysics, Max Planck Institute for Extraterrestrial Physics, New Mexico State University, New York University, Ohio State University, Pennsylvania State University, University of Portsmouth, Princeton University, the Spanish Participation Group, University of Tokyo, University of Utah, Vanderbilt University, University of Virginia, University of Washington, and Yale University. This research made use of the VizieR catalogue access tool, CDS, Strasbourg, France.

\section{References}

Ahn, C. P., Alexandroff, R., Allende Prieto, C., et al. 2012, ApJS, 203, 21 Althaus, L. G., Serenelli, A. M., Panei, J. A., et al. 2005, A\&A, 435, 631 Asplund, M., Grevesse, N., Sauval, A. J., \& Scott, P. 2009, ARA\&A, 47, 481 Barnard, A. J., Cooper, J., \& Shamey, L. J. 1969, A\&A, 1, 28

Barnard, A. J., Cooper, J., \& Smith, E. W. 1974, J. Quant. Spectr. Rad. Transf., 14,1025

\section{Bauer, F., \& Husfeld, D. 1995, A\&A, 300, 481}

Bianchi, L., Conti, A., \& Shiao, B. 2014, VizieR Online Data Catalog: II/335

Blöcker, T., \& Schönberner, D. 1996, Mem. Soc. Astron. It., 67, 665

Blöcker, T., \& Schönberner, D. 1997, A\&A, 324, 991

Bond, H. E. 2000, in Asymmetrical Planetary Nebulae II: From Origins to Microstructures, eds. J. H. Kastner, N. Soker, \& S. Rappaport, ASP Conf. Ser., 199, 115

Brown, T. M., Sweigart, A. V., Lanz, T., Landsman, W. B., \& Hubeny, I. 2001, ApJ, 562, 368

Chayer, P., Dixon, W. V., Fullerton, A. W., Ooghe-Tabanou, B., \& Reid, I. N. 2015, MNRAS, 452, 2292
Chu, Y.-H., Su, K. Y. L., Bilikova, J., et al. 2011, AJ, 142, 75

Clayton, G. C., De Marco, O., Nordhaus, J., et al. 2014, AJ, 147, 142

Cutri, R. M., \& et al. 2014, VizieR Online Data Catalog: II/328

De Marco, O. 2014, in Asymmetrical Planetary Nebulae VI Conference, 122

De Marco, O., Hillwig, T. C., \& Smith, A. J. 2008, AJ, 136, 323

De Marco, O., Farihi, J., \& Nordhaus, J. 2009, J. Phys. Conf. Ser., 172, 012031

De Marco, O., Long, J., Jacoby, G. H., et al. 2015, MNRAS, 448, 3587

Deroo, P., van Winckel, H., Min, M., et al. 2006, A\&A, 450, 181

Deroo, P., Acke, B., Verhoelst, T., et al. 2007, A\&A, 474, L45

Dimitrijević, M. S., \& Sahal-Brechot, S. 1992, A\&AS, 96, 613

Dimitrijević, M. S., Sahal-Bréchot, S., \& Bommier, V. 1991, Bulletin de

l'Observatoire Astronomique de Belgrade, 144, 65

Dreizler, S., \& Werner, K. 1996, A\&A, 314, 217

Fitzpatrick, E. L. 1999, PASP, 111, 63

Fontaine, M., Chayer, P., Oliveira, C. M., Wesemael, F., \& Fontaine, G. 2008, ApJ, 678, 394

Fujimoto, M. Y. 1977, PASJ, 29, 331

Gänsicke, B. T., Marsh, T. R., Southworth, J., \& Rebassa-Mansergas, A. 2006, Science, 314, 1908

Geier, S., Hirsch, H., Tillich, A., et al. 2011, A\&A, 530, A28

Geier, S., Kupfer, T., Heber, U., et al. 2015, A\&A, 577, A26

Gianninas, A., Bergeron, P., Dupuis, J., \& Ruiz, M. T. 2010, ApJ, 720, 581

Griem, H. R. 1974, Spectral line broadening by plasmas (New York: Academic Press), Inc. Pure and Applied Physics, 39, 421

Hajduk, M., Zijlstra, A. A., \& Gesicki, K. 2010, MNRAS, 406, 626

Han, Z., \& Webbink, R. F. 1999, A\&A, 349, L17

Heber, U., \& Hunger, K. 1987, The Messenger, 47, 36

Heber, U., \& Kudritzki, R. P. 1986, A\&A, 169, 244

Heber, U., Hunger, K., Jonas, G., \& Kudritzki, R. P. 1984, A\&A, 130, 119

Herald, J. E., \& Bianchi, L. 2011, MNRAS, 417, 2440

Herwig, F. 2001, in Astrophys. Space Sci. Lib. 265, eds. R. Szczerba, \& S. K. Górny, 249

Hirsch, H. A. 2009, Ph.D. Thesis, University Erlangen-Nürnberg

Horne, K., \& Marsh, T. R. 1986, MNRAS, 218, 761

Hügelmeyer, S. D., Dreizler, S., Homeier, D., et al. 2006, A\&A, 454, 617

Iben, Jr., I., \& Tutukov, A. V. 1984, ApJS, 54, 335

Iben, Jr., I., \& Tutukov, A. V. 1986, ApJ, 311, 742

Iben, Jr., I., Kaler, J. B., Truran, J. W., \& Renzini, A. 1983, ApJ, 264, 605

Jeffery, C. S., \& Schönberner, D. 2006, A\&A, 459, 885

Justham, S., Podsiadlowski, P., \& Han, Z. 2011, MNRAS, 410, 984

Justham, S., Podsiadlowski, P., Han, Z., \& Wolf, C. 2010, Ap\&SS, 329, 3

Kalirai, J. S. 2012, Nature, 486, 90

Kordopatis, G., Recio-Blanco, A., de Laverny, P., et al. 2011, A\&A, 535, A107

Kronberger, M., Jacoby, G. H., Harmer, D., \& Patchick, D. 2014, in Asymmetrical Planetary Nebulae VI Conference, 47

Lanz, T., Brown, T. M., Sweigart, A. V., Hubeny, I., \& Landsman, W. B. 2004, ApJ, 602, 342

Latour, M., Fontaine, G., Brassard, P., Chayer, P., \& Green, E. M. 2010, in AIP Conf. Ser. 1273, eds. K. Werner, \& T. Rauch, 255

Latour, M., Fontaine, G., Green, E. M., \& Brassard, P. 2015, A\&A, 579, A39

Lawrence, A., Warren, S. J., Almaini, O., et al. 2013, VizieR Online Data Catalog: II/319

Lemke, M. 1997, A\&AS, 122, 285

Mahsereci, M. 2011, Diploma thesis, University Tübingen, Germany

Manick, R., Miszalski, B., \& McBride, V. 2015, MNRAS, 448, 1789

Marsh, T. R., Nelemans, G., \& Steeghs, D. 2004, MNRAS, 350, 113

Maxted, P. F. L., Heber, U., Marsh, T. R., \& North, R. C. 2001, MNRAS, 326, 1391

Mendez, R. H. 1991, in Evolution of Stars: the Photospheric Abundance Connection, eds. G. Michaud, \& A. V. Tutukov, IAU Symp., 145, 375

Miller Bertolami, M. M. 2016, A\&A, in press,

DOI: $10.1051 / 0004-6361 / 201526577$

Miller Bertolami, M. M., \& Althaus, L. G. 2006, A\&A, 454, 845

Miller Bertolami, M. M., \& Althaus, L. G. 2007, A\&A, 470, 675

Miszalski, B., Acker, A., Moffat, A. F. J., Parker, Q. A., \& Udalski, A. 2009, A\&A, 496, 813

Nagel, T., Schuh, S., Kusterer, D.-J., et al. 2006, A\&A, 448, L25

Napiwotzki, R. 1999, A\&A, 350, 101

Napiwotzki, R., Yungelson, L., Nelemans, G., et al. 2004, in Spectroscopically and Spatially Resolving the Components of the Close Binary Stars, eds. R. W. Hilditch, H. Hensberge, \& K. Pavlovski, ASP Conf. Ser., 318, 402

Nelemans, G., Yungelson, L. R., van der Sluys, M. V., \& Tout, C. A. 2010, MNRAS, 401, 1347

Paczynski, B. 1976, in Structure and Evolution of Close Binary Systems, eds. P. Eggleton, S. Mitton, \& J. Whelan, IAU Symp., 73, 75

Parker, Q. A., Acker, A., Frew, D. J., et al. 2006, MNRAS, 373, 79

Piersanti, L., Tornambé, A., \& Yungelson, L. R. 2014, MNRAS, 445, 3239

Piersanti, L., Yungelson, L. R., \& Tornambé, A. 2015, MNRAS, 452, 2897 
Rauch, T., \& Deetjen, J. L. 2003, in Stellar Atmosphere Modeling, eds. I. Hubeny, D. Mihalas, \& K. Werner, ASP Conf. Ser., 288, 103

Rauch, T., Köppen, J., \& Werner, K. 1994, A\&A, 286, 543

Rauch, T., Ziegler, M., Werner, K., et al. 2007, A\&A, 470, 317

Reindl, N., Rauch, T., Werner, K., et al. 2014a, A\&A, 572, A117

Reindl, N., Rauch, T., Werner, K., Kruk, J. W., \& Todt, H. 2014b, A\&A, 566, A116

Schlafly, E. F., \& Finkbeiner, D. P. 2011, ApJ, 737, 103

Schönberner, D. 1979, A\&A, 79, 108

Schönberner, D. 2008, in Hydrogen-Deficient Stars, eds. A. Werner, \& T. Rauch, ASP Conf. Ser., 391, 139

Schöning, T., \& Butler, K. 1989, A\&AS, 78, 51

Schuh, S., Beeck, B., \& Nagel, T. 2009, J. Phys. Conf. Ser., 172, 012065

Schwarz, C., Montgomery, M. M., \& Martin, E. L. 2010, in AIP Conf. Ser. 1273, eds. K. Werner, \& T. Rauch, 362

Shimansky, V. V., Borisov, N. V., Nurtdinova, D. N., et al. 2015, Astron. Rep., 59, 199

Stroeer, A., Heber, U., Lisker, T., et al. 2007, A\&A, 462, 269

Tremblay, P.-E., \& Bergeron, P. 2009, ApJ, 696, 1755

van Winckel, H., Lloyd Evans, T., Briquet, M., et al. 2009, A\&A, 505, 1221

Verbeek, K., Groot, P. J., Scaringi, S., et al. 2014, MNRAS, 438, 2 von Hippel, T., Kuchner, M. J., Kilic, M., Mullally, F., \& Reach, W. T. 2007, ApJ, 662, 544

Wassermann, D., Werner, K., Rauch, T., \& Kruk, J. W. 2010, A\&A, 524, A9

Webbink, R. F. 1984, ApJ, 277, 355

Werner, K., \& Herwig, F. 2006, PASP, 118, 183

Werner, K., \& Rauch, T. 2014, A\&A, 569, A99

Werner, K., Deetjen, J. L., Dreizler, S., et al. 2003, in Stellar Atmosphere Modeling, eds. I. Hubeny, D. Mihalas, \& K. Werner, ASP Conf. Ser., 288, 31

Werner, K., Rauch, T., \& Kepler, S. O. 2014, A\&A, 564, A53

Wright, E. L., Eisenhardt, P. R. M., Mainzer, A. K., et al. 2010, AJ, 140, 1868

Xu, S., \& Jura, M. 2012, ApJ, 745, 88

Young, P., Schneider, D. P., \& Shectman, S. A. 1981, ApJ, 245, 1035

Yuan, H. B., \& Liu, X. W. 2013, MNRAS, 436, 718

Yungelson, L. R., Nelemans, G., \& van den Heuvel, E. P. J. 2002, A\&A, 388, 546

Zhang, X., \& Jeffery, C. S. 2012a, MNRAS, 426, L81

Zhang, X., \& Jeffery, C. S. 2012b, MNRAS, 419, 452

Zhang, X., Jeffery, C. S., Chen, X., \& Han, Z. 2014, MNRAS, 445, 660

Ziegler, M. 2012, Dissertation, University of Tübingen, Germany, https:// publikationen. uni-tuebingen.de/xmlui/handle/10900/50006 